\title{
The Optimization Design of PID Controller Parameters Based On Particle Swarm Optimization
}

\author{
Zhaosheng LI \\ North China Electric Power University, Baoding 071000, China \\ 1170188363@qq.com
}

Keywords: Particle Swarm Optimization; PID controller; ITAE

\begin{abstract}
In recent years, automatic control system have assumed an increasingly important role in the development and advancement of modern civilization and technology. PID controller is the most popular in the automatic system. The setting and optimization of PID parameters are always the important study topics in the automatic control field. The control effect depends on proportion, differential and integral which are the parameters of PID controller. Original optimization method is a time-consuming method and cannot get satisfied control effect. In order to solve this problem, Particle Swarm Optimization (PSO) is applied to PID controller. Establish a PID controller parameters model based on PSO, in which PID parameters act as particle in the particle swarm, using minimizing integral of time-weighted absolute error (ITAE) as the optimization objective function, thereby performing PID control parameters optimization.
\end{abstract}

\section{Introduction}

The PID controller is the most common form of feedback. It was an essential element of early governors and it became the standard tool when process control emerged in the 1940s. In process control today, more than $95 \%$ of the control loops. PID controllers have survived many changes in technology, from mechanics and pneumatics to microprocessors via electronic tubes, transistors, integrated circuits. The PID algorithm is described by:

$$
u(t)=K_{p} e(t)+K_{i} \int_{0}^{t} e(\tau) d \tau+K_{d} \frac{d e(t)}{d t}
$$

where $u$ is the control signal, $e$ is the control error, $K_{p} 、 K_{i}$ and $K_{d}$ are the weights of the error the integral of the error and the derivative of the error. The controller is able to calculate the control signal based on different weights, which can drive the controlled object. If the controller design is reasonable, the error of the control signal will reduce and meet the control requirements.

Therefore, Optimizing PID controller parameters is significant. PID controller parameters are mainly adjusted by people, which not only consumes much time, but also cannot guarantee optimum performance. PSO has been widely used in the optimization of function、 neural networks 、 pattern classification and other applications. In this case, we will design PID controller parameters by using PSO.

\section{Problem Description:}

The block diagram of the PID control system is shown in Fig.1, where $r, e, y$ are respectively the reference, error and controlled variables.

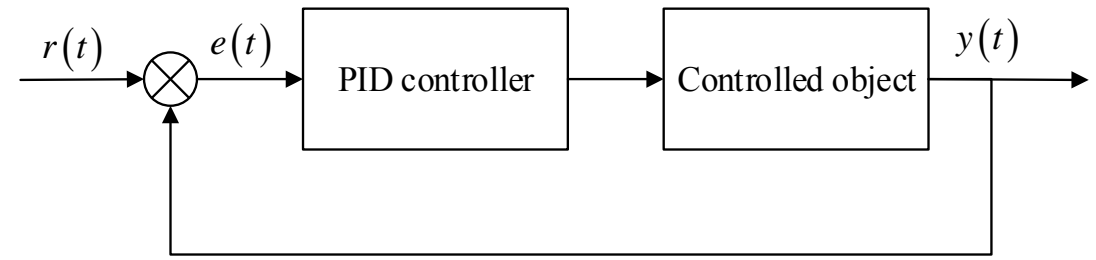

Fig.1 The block diagram of PID control system 
Furthermore, performance index is defined as a quantitative measure to depict the system performance of the designed PID controller. Using this technique an 'optimum system' can often be designed and a set of PID parameters in the system can be adjusted to meet the required specification. For a PID-controlled system, there are often four indices to depict the system performance: ISE、IAE、ITAE and ITSE. They are defined as follows:

$$
\begin{aligned}
& I S E=\int_{0}^{\infty} e^{2}(t) d t \\
& \text { ITAE }=\int_{0}^{\infty} t|e(t)| d t \\
& I A E=\int_{0}^{\infty}|e(t)| d t \\
& \text { ITSE }=\int_{0}^{\infty} t e^{2}(t) d t
\end{aligned}
$$

Where $t$ is the time and $e(t)$ is the difference between set point and controlled variable. ITAE is used to describe the performance for different parameters in this case.

The controlled object transfer function is

$$
G(s)=\frac{s+2}{s^{4}+8 s^{3}+4 s^{2}-s+0.4}
$$

The model established in the Simulink environment are shown in Fig.2.

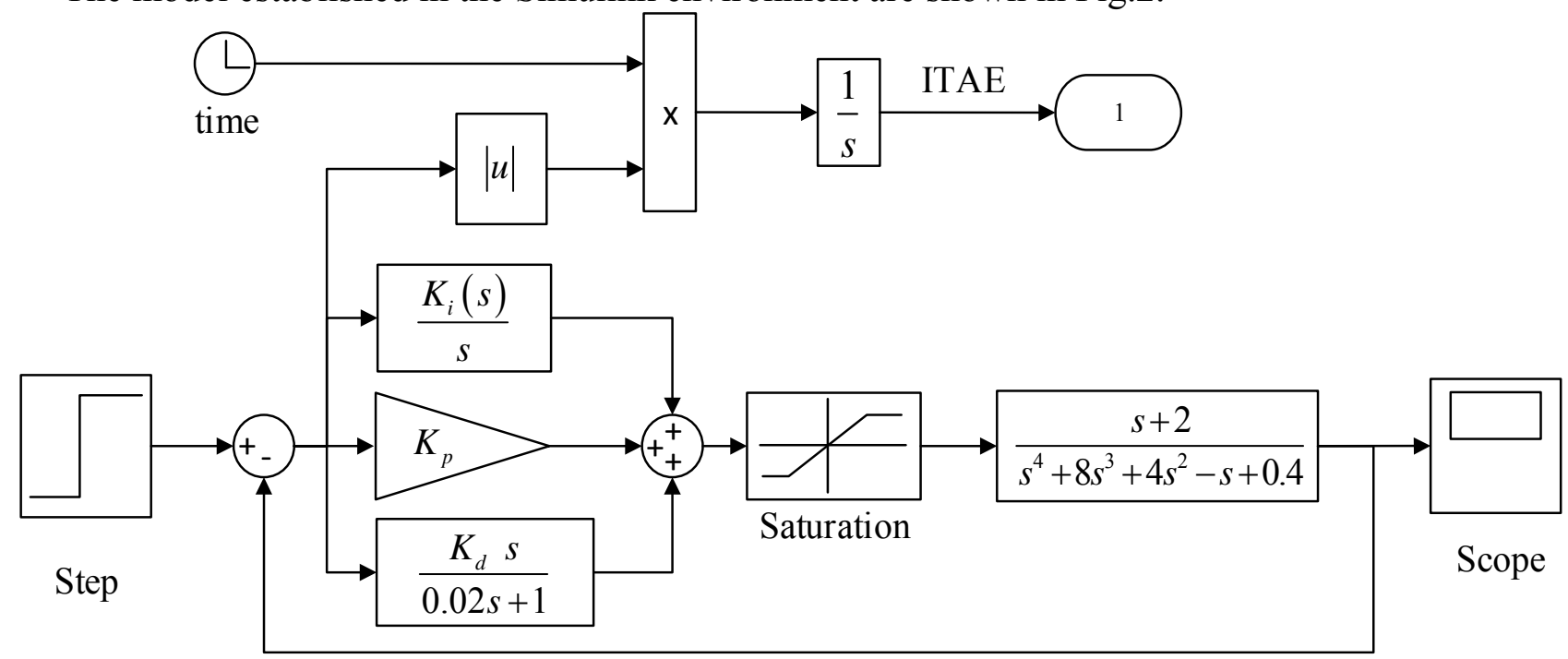

Fig.2 Simulink model

In the Fig.2, the differential link is replaces by fist-order link, the output port is ITAE index which is obtained by the integration of the product (time and absolute error).

\section{Optimum Design Process}

Optimize the parameters of PID controller based on PSO algorithm, the process shown in Fig.3. 


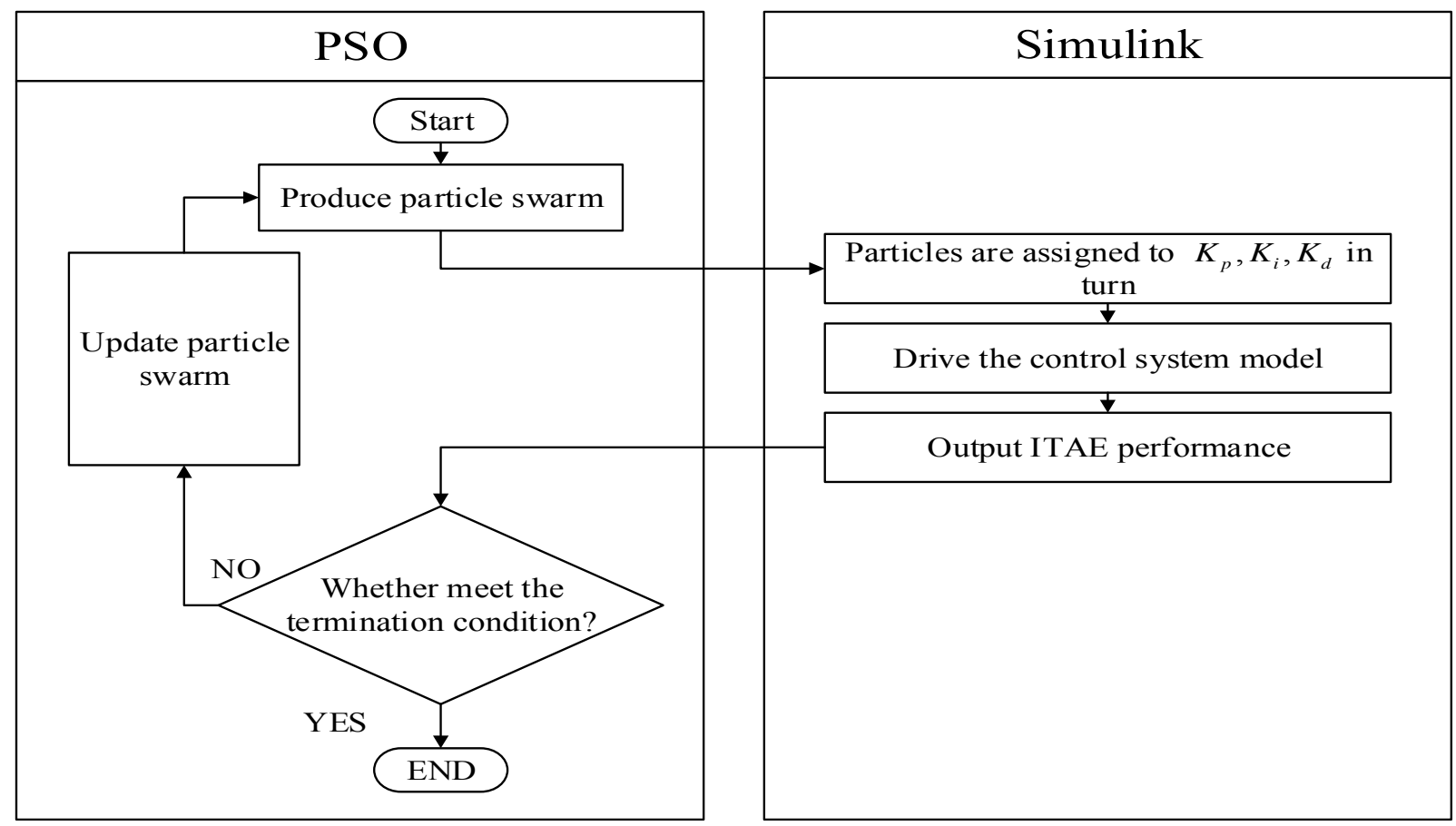

Fig.3 The process of optimization

In Fig.3, the bridges of connecting PSO algorithm to Simulink model are particles (PID controller parameters) and fitness values of corresponding particles (the performance of control system).

\section{Achieve PSO algorithm}

PSO is optimization algorithm based on evolutionary computer technique. The basic PSO is developed from research on swarm such as fish schooling and bird flocking ${ }^{[1]}$. After it was firstly introduced in $1995^{[2]}$, a modified PSO was then introduced in 1998 to improve the performance of the original PSO. Velocity and position of the particle in the search space is determined by the following formula.

$$
\begin{gathered}
v_{t+1}=w v_{t}+c_{1} r_{1}\left(P_{t}-x_{t}\right)+c_{2} r_{2}\left(G_{t}-x_{t}\right) \\
x_{t+1}=x_{t}+v_{t+1}
\end{gathered}
$$

Where $C_{1}$ and $C_{2}$ are two positive constant. As recommended, the constant are $C_{1}=C_{2}=2$. While $r$ is random function between 0 and 1 , and $t$ represents iteration. The best previous position (giving the minimum fitness value) of any particle is recorded and represented as $P$, this is called Pbest. The index of the best particle among all particles in the population is represented by the symbol $G$, called as Gbest. Inertia weight, $w$ is brought into the equation to balance between the global search and local search capability ${ }^{[3]}$.The PSO process ${ }^{[4]}$ is as follows.

- Specify the lower and upper bounds of the three controller parameters and initialize randomly the individuals of the population including searching points、velocities、Gbest and Pbest.

- Compare fitness value of the current particle with $P_{t}$ of experiencing the entire particle swarm. If the current value is better, it will act as current $P_{t}$.

- Compare fitness value of the current particle with $G_{t}$ of experiencing the entire particle swarm. If the current value is better, it will act as current $G_{t}$.

- Update the velocities and position of the particle according to formula (7) and (8).

- If not meeting the termination condition (usually the default number of iterations and the fitness value of the lower limit), return to the step (2). Otherwise, exit the algorithm and obtain the optimal solution. 


\section{Result Anaysis}

Program by using MATLAB, and get the following optimal process. The performance is shown in Fig.4. The changing process of $K_{p} 、 K_{i}$ and $K_{d}$ are shown in Fig.5.

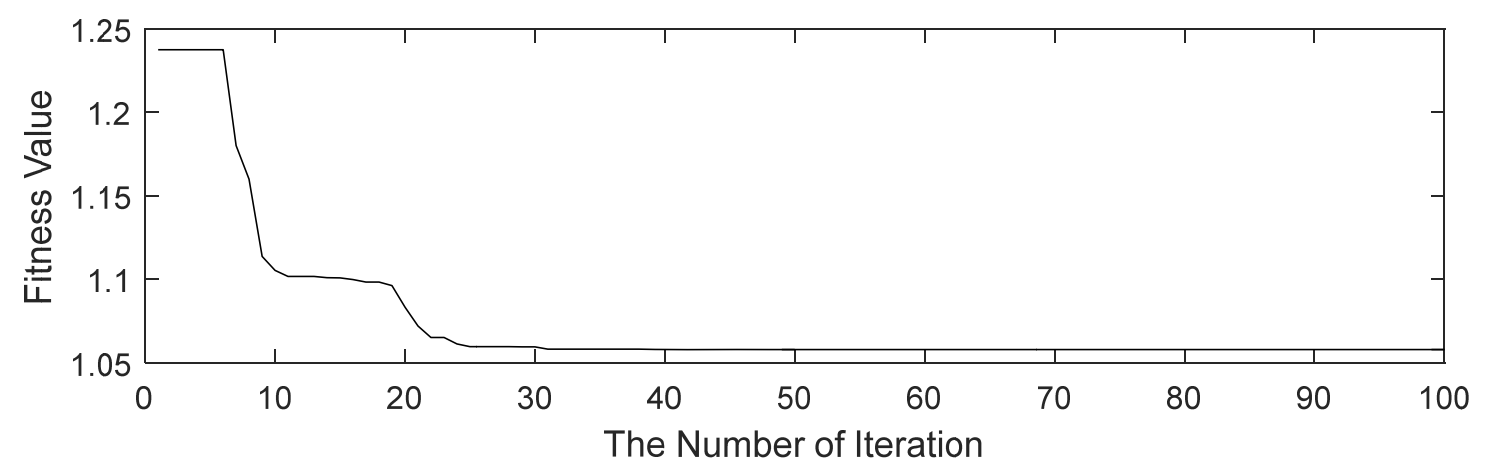

Fig.4 The best fitness value
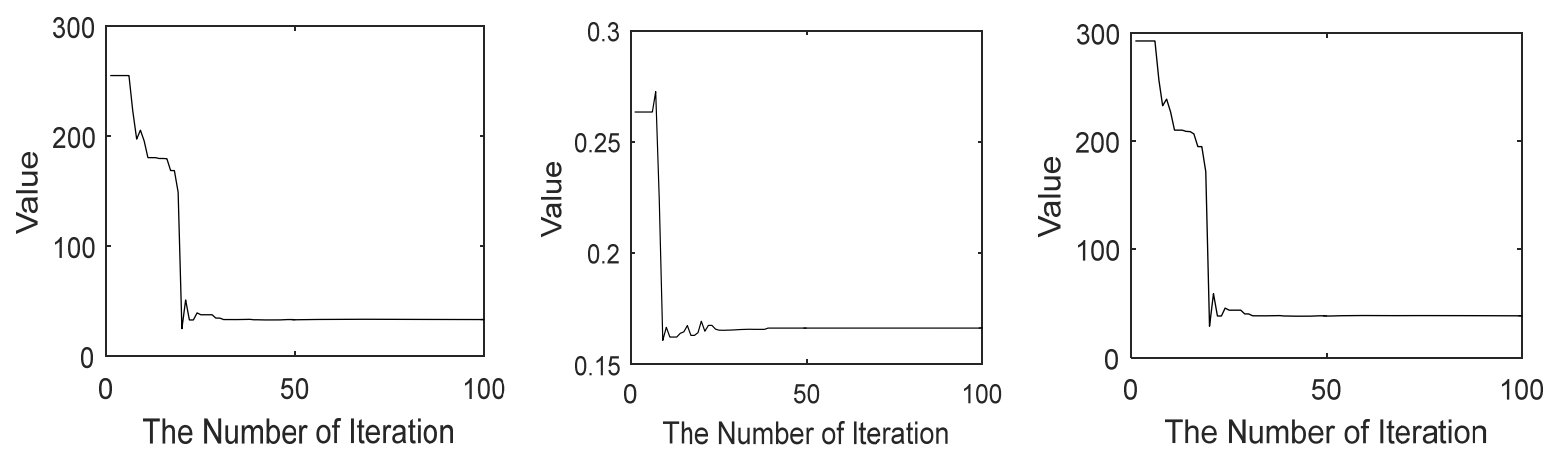

Fig.5 Optimal cure of $\mathrm{Kp} 、 \mathrm{Ki} 、 \mathrm{Kd}$

Get the following result according to the Fig.4 and Fig.5.

$$
\begin{aligned}
& K_{p}=33.4250, K_{i}=0.1663 \\
& K_{d}=38.5447, \quad \text { ITAE }=1.0580
\end{aligned}
$$

The step response figure is shown in Fig. 6 according to above parameters.

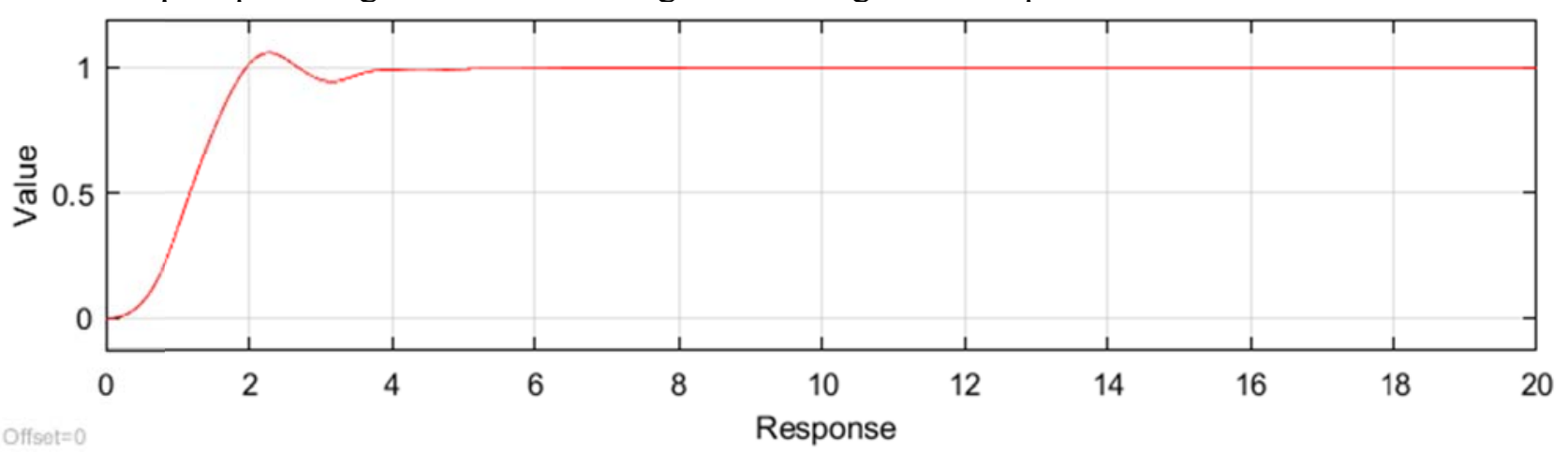

Fig. 6 The unit step response curve for the best parameters

\section{References}

[1] Ou, C. and Lin, W. (2006). Comparison between PSO and GA for parameters optimization of PID controller. Proc. IEEE International Conference on Mechatronics and Automation. Luoyang, China.

[2] Kennedy, J. and Eberhart, R.C. (1995). Particle swarm optimization. Proc. IEEE International 
Conference on Neural Networks (Perth, Australia), IEEE Service Center, Piscataway, NJ, pp. IV: 1942- 1948.

[3] Shi, Y.H. and Eberhart, R.C. (1998). A modified particle swarm optimizer. IEEE International Conference on Evolutionary Computation, Anchorage, Alaska.

[4] Zwe-Lee Gaing. (2004). A Particle Swarm Optimization Approach for Optimum Design of PID Controller in AVR system. IEEE transactions on energy conversion. 